\title{
Lefschetz coincidence formula on non-orientable manifolds
}

\author{
by \\ Daciberg Lima Gonçalves (São Paulo) \\ and Jerzy J e z i ers ki (Warszawa)
}

\begin{abstract}
We generalize the Lefschetz coincidence theorem to non-oriented manifolds. We use (co-) homology groups with local coefficients. This generalization requires the assumption that one of the considered maps is orientation true.
\end{abstract}

1. Introduction. The Lefschetz Fixed Point Theorem may be reformulated to detect coincidences of a pair of maps $f, g: M \rightarrow N$ between closed manifolds of the same dimension ([V], Ch. 6, [Bd], Ch. 6). Such a modification, however, needs the orientability assumption on $M$ and $N$. In the same chapter, page 176 of [V], the author says "It should be pointed out the spaces we consider, closed, orientable manifolds, could be made more general. Similar techniques may be applied in the nonorientable case by using twisted coefficients." The aim of this work is to present such an extension. So we drop the assumption that the manifolds are orientable and we only assume that one of the considered maps, say $g$, is orientation true, i.e. a loop $\alpha$ preserves a local orientation of $M$ iff $g \alpha$ preserves a local orientation of $N$. However, without the above assumption on $g$ such an extension does not seem possible.

In Section 2 we prepare some information on (co-) homology with coefficients in a local system. We use them (Section 3) to prove the Poincaré duality and then to get the promised Lefschetz theorem (Section 4). In Section 5 we find a relation between the coincidence index defined there and the semi-index from $[\mathrm{DJ}]$ and $[\mathrm{Je}]$. At the end of this section we sketch how to define an index for a Nielsen class without restrictions on the maps $f$ and $g$. In Section 6 we prove a result about coincidence producing maps as defined in $[\mathrm{BS}]$.

1991 Mathematics Subject Classification: Primary 55M20; Secondary 57N99.

The first author has been supported by the international cooperation program GMD/Germany-Cnpq/Brasil and Universidade de São Paulo during his visit at the Mathematische Institut der Universität Heidelberg, where part of this work was done. 
2. Homology with local coefficients. In this section we recall some basic information about homology with coefficients in a local system [Wh], [Sp2]. Let $R$ be a commutative ring with unity, $X$ a topological space and let $\Gamma$ be a local system of $R$-modules over $X$, i.e. over each point $x \in X$ we have a module $\Gamma(x)$ and for each homotopy class $\omega$ of paths from $x_{0}$ to $x_{1}$ we have an $R$-module homomorphism $\Gamma(\omega): \Gamma\left(x_{0}\right) \rightarrow \Gamma\left(x_{1}\right)$. Let $\Delta_{*}(X ; \Gamma)$ denote the chain complex with coefficients in $\Gamma$ (i.e. the graded $R$-module of sums $\sum \gamma_{\sigma} \cdot \sigma$ where $\sigma$ is a singular simplex in $X$ with $\gamma_{\sigma} \in$ $\Gamma\left(\sigma\left(v_{0}\right)\right)$, where $v_{0}$ denotes the leading vertex of the standard $n$-simplex) and let $H_{*}(X ; \Gamma)$ be its homology module obtained by using the boundary operators defined on local coefficients. Similarly we consider the cohomology $H^{*}\left(X ; \Gamma^{*}\right)$ with coefficients in the dual system $\Gamma^{*}=\operatorname{Hom}(\Gamma, R)$ (see [Wh], [Sp2] for details). Let $\Gamma, \Gamma^{\prime}$ be local systems over $X$ and $Y$ respectively. To get a chain homomorphism induced by a map $f: X \rightarrow Y$ we need a morphism of the given local systems, i.e. a commutative diagram

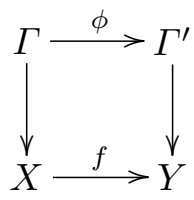

where the restriction of $\phi$ to any fibre is a homomorphism and

$$
\phi(\omega(1)) \cdot \Gamma(\omega)=\Gamma^{\prime}(f \omega) \cdot \phi(\omega(0))
$$

for any path $\omega$ in $X$, where $\phi(x)$ is the homomorphism $\phi$ restricted to the fibre $\Gamma(x)$. We define maps $f_{*}: \Delta_{*}(X ; \Gamma) \rightarrow \Delta_{*}\left(Y ; \Gamma^{\prime}\right)$ by $f_{*}(\gamma \cdot \sigma)=$ $\phi(\gamma) \cdot f \sigma$ and $f^{*}: \Delta^{*}\left(Y ; \Gamma^{\prime *}\right) \rightarrow \Delta^{*}\left(X ; \Gamma^{*}\right)$ by $\left(f^{*}(c)(\sigma)\right)(\gamma)=(c(f \sigma))(\phi \gamma)$.

Recall that there is a natural pairing (Kronecker index)

$$
\langle\cdot, \cdot\rangle: H^{q}\left(X, A ; \Gamma^{*}\right) \otimes H_{q}(X, A ; \Gamma) \rightarrow R .
$$

The symbol $\otimes$ denotes tensor product over $R$. We will also denote by $R$ the constant system with the fibre $R$.

In this paper $\Gamma, \Gamma^{\prime}, \ldots$ denote one-dimensional local systems, i.e. each $\Gamma(x)$ is an $R$-module isomorphic to $R$. Then the chain complexes $\Delta_{*}(X \times Y$; $\left.\Gamma \times \Gamma^{\prime}\right)$ and $\Delta(X, \Gamma) \otimes \Delta\left(Y, \Gamma^{\prime}\right)$ are naturally isomorphic. This yields the following products:

- the cross product

$$
\begin{aligned}
\times: H_{p}(X, A ; \Gamma) \otimes H_{q}\left(Y, B ; \Gamma^{\prime}\right) & \rightarrow H_{p+q}\left((X, A) \times(Y, B) ; \Gamma \times \Gamma^{\prime}\right), \\
\times: H^{p}\left(X, A ; \Gamma^{*}\right) \otimes H^{q}\left(Y, B ; \Gamma^{\prime *}\right) & \rightarrow H^{p+q}\left((X, A) \times(Y, B) ;\left(\Gamma \times \Gamma^{\prime}\right)^{*}\right),
\end{aligned}
$$

(provided $X \times B, A \times Y$ is excisive in $X \times Y$ ), 
- the cup product

$\cup: H^{p}\left(X, A ; \Gamma^{*}\right) \otimes H^{q}\left(X, B ; \Gamma^{*}\right) \rightarrow H^{p+q}\left(X, A \cup B ;\left(\Gamma \otimes \Gamma^{\prime}\right)^{*}\right)$,

- the cap product

$$
\cap: H^{p}(X, A ; G) \otimes H_{n}(X, A \cup B ; \Gamma) \rightarrow H_{n-p}(X, B ; \Gamma \otimes G)
$$

(in the cup and cap products we assume that $\{A, B\}$ is excisive in $A \cup B$ ).

For future reference we list some of their properties:

2.1. Let $\Gamma, \Gamma^{\prime}$ be local systems on $X$ and let $x \in H^{p}\left(X, A ; \Gamma^{*}\right)$, $y \in H^{q}\left(X, B ; \Gamma^{* *}\right)$. Then

$$
x \cup y=(-1)^{p q}(y \cup x) .
$$

2.2. Let $x \in H^{p}\left(X, A ; \Gamma^{*}\right), y \in H^{q}(X ; R)$ and $a \in H_{p+q}(X, A ; \Gamma)$. Then

$$
\langle x, y \cap a\rangle=\langle x \cup y, a\rangle .
$$

2.3. Let $x \in H^{p}(X, A ; R), y \in H^{q}\left(Y, A^{\prime} ; R\right), a \in H_{r}(X, A \cup B ; \Gamma)$ and $b \in H_{s}\left(Y, A^{\prime} \cup B^{\prime} ; \Gamma^{\prime}\right)$. Then

$$
\begin{aligned}
(x \times y) \cap(a \times b)=(-1)^{p(s-q)} & (x \cap a) \times(y \cap b) \\
& \in H_{r+s-p-q}\left((X, B) \times\left(Y, B^{\prime}\right) ; \Gamma \times \Gamma^{\prime}\right) .
\end{aligned}
$$

In the next section we will also use the following lemma (compare the formula (5.20) on p. 150 of [V]).

LEMma 2.4. Let $M$ be an $n$-manifold with a compact boundary and fix $U \in H^{n}\left(M \times M, M \times M-\Delta ;(R \times \Gamma)^{*}\right)$ and $x \in H^{p}(M, \partial M ; R)$. Let $\widetilde{U} \in H^{n}\left(M \times M, \partial(M \times M)-\partial \Delta ;(R \times \Gamma)^{*}\right)$ be the restriction of $U$ and let $1 \in H^{0}(M ; R)$ denote the unit. Then

$$
\widetilde{U} \cup(x \times 1)=\widetilde{U} \cup(1 \times x) \in H^{n+p}\left(M \times M, \partial(M \times M) ;(R \times \Gamma)^{*}\right) .
$$

Proof. It is enough to show that $U \cup(x \times 1)=U \cup(1 \times x) \in H^{n+p}(M \times$ $\left.M, M \times M-\dot{\Delta}) ;(R \times \Gamma)^{*}\right)$ (here $\left.\dot{\Delta}=\Delta-\partial \Delta\right)$. Let us fix:

(i) a collar $C=\partial M \times[0,1) \subset M$;

(ii) a neighbourhood $V$ of $\Delta \subset M \times M$ such that the projections $p_{1}, p_{2}$ : $V \rightarrow M$ are homotopic rel. $\Delta\left(p_{i}\left(x_{1}, x_{2}\right)=x_{i}\right)$;

(iii) a neighbourhood $V_{0}$ of $\partial \Delta \subset M \times M$ such that $V_{0} \subset V \cap(C \times$ $C), p_{1}\left(V_{0}\right) \cup p_{2}\left(V_{0}\right) \subset C$ and the above homotopy is a homotopy of pairs $\left(V, V_{0}\right) \rightarrow(M, C)$

(iv) a collar $C_{1}$ of $\partial \Delta \subset \Delta$ contained in $V_{0}$. 
Set $\Delta_{1}=\Delta-C_{1}$. Consider the commutative diagram

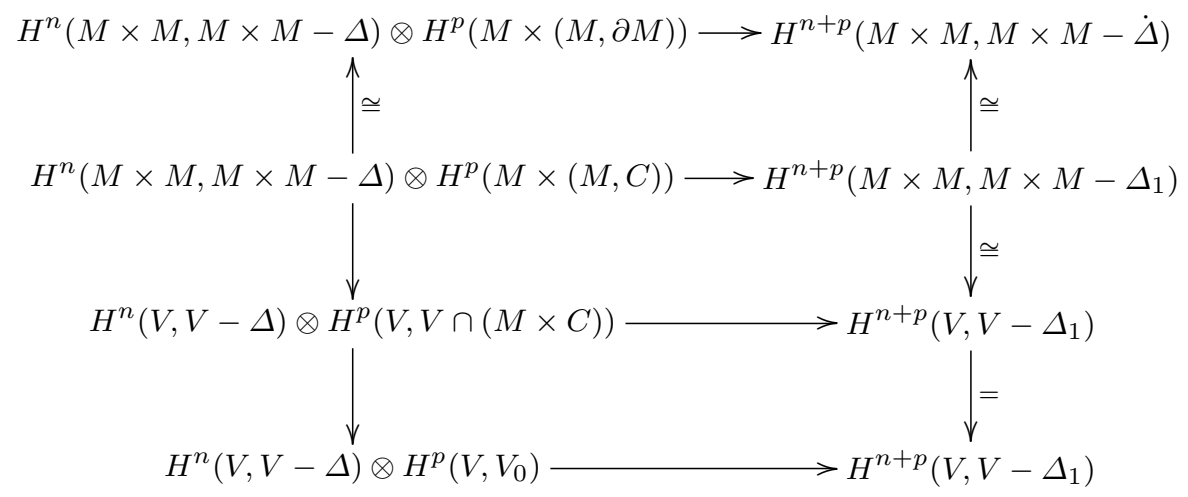

where the horizontal arrows are cup products, the vertical arrows are induced by the natural inclusions and the coefficients in any line are $(R \times \Gamma)^{*} \otimes(R \times R) \rightarrow(R \times \Gamma)^{*}$. Consider the cup product $U \cup(1 \times x)$ in the top line. In the bottom line we get $U_{\mid V} \cup(1 \times x)_{\mid V}$ (here we denote by $U_{\mid V}$ the image of $U$ under the natural homomorphism $H^{n}(M \times$ $\left.M, M \times M-\Delta ;\left(R \times \Gamma^{*}\right)\right) \rightarrow H^{n}\left(V, V-\Delta ;\left(R \times \Gamma^{*}\right)\right)$ and we identify $\left.x \in H^{p}(M, \partial M)=H^{p}(M, C)\right)$. Considering the similar diagram for the cup product $U \cup(x \times 1)$, in the bottom line we obtain $U_{\mid V} \cup(x \times 1)_{\mid V}$. But

$$
(1 \times x)_{\mid V}=\left(p_{2}^{*} x\right)_{\mid V}=\left(p_{1}^{*} x\right)_{\mid V}=(x \times 1)_{\mid V} \in H^{p}\left(V, V_{0}\right)
$$

since $p_{1 \mid V}, p_{2 \mid V}:\left(V, V_{0}\right) \rightarrow(M, C)$ are homotopic. Since the right sides of both diagrams are identical and consist of isomorphisms,

$$
(1 \times x)_{\mid V}=(x \times 1)_{\mid V} \in H^{n+p}\left(V, V-\Delta_{1}\right)
$$

implies

$$
U \cup(1 \times x)=U \cup(x \times 1) \in H^{n+p}(M \times M, M \times M-\dot{\Delta}) .
$$

3. Poincaré duality. In this section we prove the Poincaré Duality Theorem for a pair $(K, L)$ of compact subspaces of a (non-orientable) manifold, where the Poincaré map goes from Cech cohomology of a pair to homology by taking the cap product with the fundamental class. This is the form of the duality that we need in the proof of the Lefschetz coincidence theorem. The Poincaré Duality Theorem is also proved in [Sp2] (a Poincaré map is defined by slant product and goes from homology to cohomology) and in [W1], [W2] (for any local coefficient system and some pairs of subspaces). In our case we will modify Section VI of [B] for the non-orientable case. Finally, we define the Thom class in the same fashion as in the orientable case and consider the case of a manifold with boundary. Let us point out that the Thom class can also be defined using duality as in [G1]. 
Let $M$ be a topological $n$-manifold without boundary. In the rest of this paper $\Gamma_{M}$ will denote the orientation system on $M: \Gamma_{M}(x)=H_{n}(M$, $M-x ; R)$. Then for any $x \in M$ the module $H_{n}\left(M, M-x ; \Gamma_{M}\right)$ has the canonical generator $z_{M, x}$ corresponding in

$$
H_{n}\left(M, M-x ; \Gamma_{M}\right)=H_{n}(M, M-x ; \mathbb{Z}) \otimes_{\mathbb{Z}} H_{n}(M, M-x ; R)
$$

to $\gamma \otimes \gamma^{\prime}$ where $\gamma$ is a generator of $H_{n}(M, M-x ; \mathbb{Z})=\mathbb{Z}$ and $\gamma^{\prime}$ is its image in $H_{n}(M, M-x ; R)$ under the map induced by the unique ring homomorphism $\mathbb{Z} \rightarrow R$.

Lemma 3.1 (fundamental class). For any compact set $A \subset M$ there exists a unique element $z_{M, A} \in H_{n}\left(M, M-A ; \Gamma_{M}\right)$ such that for any $x \in A$ the natural homomorphism $H_{n}\left(M, M-A ; \Gamma_{M}\right) \rightarrow H_{n}\left(M, M-x ; \Gamma_{M}\right)$ sends $z_{M, A}$ to $z_{M, x}$.

Proof. It is enough to check the conditions (i)-(iii) of the Bootstrap Lemma ([Bd; VI, 7.9]).

Now we can follow Chapter VI of [Bd] to reformulate the Poincaré Duality Theorem for the non-orientable case.

For closed subsets $L \subset K \subset M$ we denote by

$$
\check{H}^{p}(K, L ; G)=\underset{\lim }{\longrightarrow}\left\{H^{p}(U, V ; G):(U, V) \supset(K, L), U, V \text { open in } M\right\}
$$

the Čech cohomology modules. Let $(K, L) \subset(U, V)$ be as above. Then there is a cap product

$$
\begin{aligned}
& \Delta^{p}(U, V ; G) \otimes\left[\left(\Delta_{n}(V ; \Gamma)+\Delta_{n}(U-L ; \Gamma)\right) / \Delta_{n}(U-K ; \Gamma)\right] \\
& \stackrel{\cap}{\rightarrow} \Delta_{n-p}(U-L, U-K ; \Gamma \otimes G)
\end{aligned}
$$

given by $f \cap(b+c)=f \cap b+f \cap c=f \cap c$. But

$$
\begin{aligned}
H_{*}\left(\left(\Delta_{*}(V ; \Gamma)+\Delta_{*}(U-L ; \Gamma)\right) / \Delta_{*}(U-K ; \Gamma)\right) & =H_{*}(U, U-K ; \Gamma \otimes G) \\
& =H_{*}(M, M-K ; \Gamma \otimes G)
\end{aligned}
$$

since $\{V, U-L\}$ is an open cover of $U$. Thus we get a cap product

$$
H^{p}(U, V ; G) \otimes H_{n}\left(M, M-K ; \Gamma_{M}\right) \rightarrow H_{n-p}(M-L, M-K ; \Gamma \otimes G) .
$$

Thus capping with $z_{M, K} \in H_{n}\left(M, M-K ; \Gamma_{M}\right)$ we get a homomorphism

$$
\cap z_{M, K}: H^{p}(U, V ; G) \rightarrow H_{n-p}(M-L, M-K ; \Gamma \otimes G)
$$

which is compatible with the inclusion of $(U, V)$. Finally, in the direct limit we get the duality map

$$
D_{K, L}: \check{H}^{p}(K, L ; G) \rightarrow H_{n-p}(M-L, M-K ; \Gamma \otimes G) .
$$

Theorem 3.2 (Poincaré duality). Let $M$ be an $n$-manifold and let $K \supset L$ be compact subsets of $M$. Then the cap product map $D_{K, L}$ : $\check{H}^{p}(K, L ; G) \rightarrow H_{n-p}(M-L, M-K ; \Gamma \otimes G)$ is an isomorphism. 
P r o o f. Modify Lemmas 8.1, 8.2 and then follow the proof of Theorem 8.3 of Chapter VI in [Bd].

Now let $M$ be a compact manifold with boundary. We will denote by $\Gamma_{M}$ the unique extension of the local system $\Gamma_{\operatorname{int} M}$ onto $M$. Adding an open collar $C$ to $\partial M$ we get a manifold without boundary $M^{\prime}=M \cup C$. Applying Theorem 3.2 to $M^{\prime}$ with $K=M$ and $L=\emptyset$ we get

Corollary 3.3. Let $M$ be a compact manifold. Then the cap product $\cap z_{M, \emptyset}: H^{p}(M ; G) \rightarrow H_{n-p}\left(M^{\prime}, M^{\prime}-M ; \Gamma_{M^{\prime}} \otimes G\right)=H_{n-p}\left(M, \partial M ; \Gamma_{M} \otimes G\right)$ is an isomorphism.

The element $z_{M} \in H_{n}\left(M, \partial M ; \Gamma_{M}\right)$ corresponding to $z_{M, \emptyset} \in H_{n}\left(M^{\prime}\right.$, $M^{\prime}-M ; \Gamma_{M}^{\prime}$ ) (by the excision isomorphism) will be called the fundamental class of the manifold $M$ with boundary. In the rest of this paper we put $G=R$. Then the isomorphism $H^{p}(M ; R) \rightarrow H_{n-p}\left(M, \partial M ; \Gamma_{M}\right)$ from Corollary 3.3 will be denoted by $D_{M}(x)=x \cap z_{M}$.

LEMMA 3.4 (Thom class; [Sp2; 4.7]). Let $M$ be a manifold without boundary. There exists a unique element $U_{M} \in H^{n}(M \times M, M \times M-\Delta$; $\left.\left(R \times \Gamma_{M}\right)^{*}\right)$ whose restriction to any fibre is dual to the fundamental class, i.e. $\left\langle\left(j_{x}\right)^{*} U_{M}, z_{M, x}\right\rangle=1$ where $j_{x}:(M, M-x) \rightarrow(M \times M, M \times M-\Delta)$ is given by $j_{x}(y)=(x, y)$.

Let $\widetilde{U}_{M}$ denote the image of $U_{M}$ under the natural homomorphism $k^{*}: H^{n}\left(M \times M, M \times M-\Delta ;\left(R \times \Gamma_{M}\right)^{*}\right) \rightarrow H^{n}\left(M \times M ;\left(R \times \Gamma_{M}\right)^{*}\right)$.

Corollary 3.5. If $M$ is closed then $\left\langle\widetilde{U}_{M}, 1 \times z_{M}\right\rangle=1$.

Pr o of. Consider the commutative diagram

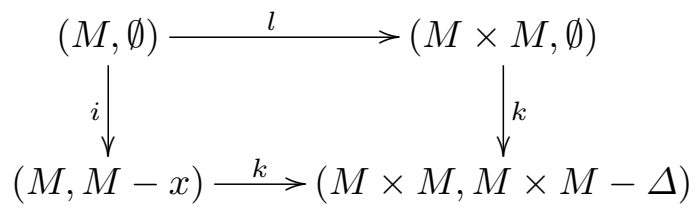

where $l(y)=j(y)=(x, y), x \in M$ fixed. Now

$$
\begin{aligned}
\left\langle\widetilde{U}_{M}, 1 \times z_{M}\right\rangle & =\left\langle k^{*}\left(U_{M}\right), l_{*}\left(z_{M}\right)\right\rangle=\left\langle l^{*} k^{*}\left(\widetilde{U}_{M}\right), z_{M}\right\rangle=\left\langle(k l)^{*}\left(\widetilde{U}_{M}\right), z_{M}\right\rangle \\
& =\left\langle(j i)^{*}\left(\widetilde{U}_{M}\right), z_{M}\right\rangle=\left\langle j^{*}\left(\widetilde{U}_{M}\right), i_{*}\left(z_{M}\right)\right\rangle=\left\langle j^{*}\left(\widetilde{U}_{M}\right), z_{M, x}\right\rangle=1 .
\end{aligned}
$$

Lemma 3.6. The inclusion $(M \times M-\partial \Delta, M \times M-\Delta) \subset(M \times M$, $M \times M-\Delta)$ is a homotopy equivalence.

Proof. It is enough to find a deformation $H:(M \times M, M \times M-\Delta) \times$ $I \rightarrow(M \times M, M \times M-\Delta)$ satisfying $H_{0}=$ identity and $H_{t}(M \times M) \subset$ $M \times M-\partial \Delta, t \in(0,1]$. Then $H_{1}:(M \times M, M \times M-\Delta) \subset(M \times M-\partial \Delta$, 
$M \times M-\Delta)$ is a homotopy inverse to our inclusion. First we define a homotopy $h: \mathbb{R}_{+} \times \mathbb{R}_{+} \times I \rightarrow \mathbb{R}_{+} \times \mathbb{R}_{+}$by

$$
h(t, s, \tau)= \begin{cases}(t+(1-t-s) \tau / 2, s+(1-t-s) \tau / 2) & \text { for } t+s \leq 1, \\ (t, s) & \text { for } t+s \geq 1 .\end{cases}
$$

This homotopy shifts $\mathbb{R}_{+} \times \mathbb{R}_{+}$into $\mathbb{R}_{+} \times \mathbb{R}_{+}-\{(0,0)\}$. Now we fix a collar $\partial M \times \mathbb{R}_{+}$in the manifold $M$, identify $\partial M \times \mathbb{R}_{+} \times \partial M \times \mathbb{R}_{+}=$ $\partial M \times \partial M \times \mathbb{R}_{+} \times \mathbb{R}_{+}$and define a deformation of $\partial M \times \partial M \times \mathbb{R}_{+} \times \mathbb{R}_{+}$ by $H(x, y, t, s, \tau)=(x, y, h(t, s, \tau \max (0,1-d(x, y)))$. Since the carrier of this homotopy is locally compact, it extends (by identity) to the desired deformation of $M \times M$.

Corollary 3.7. The inclusion (int $M \times \operatorname{int} M$, int $M \times \operatorname{int} M-\Delta) \subset$ $(M \times M, M \times M-\Delta)$ induces a (co-) homology group isomorphism.

Proof. The inclusion (int $M \times \operatorname{int} M$, int $M \times \operatorname{int} M-\Delta) \subset(M \times M$ $-\partial \Delta, M \times M-\Delta)$ is excisive and $(M \times M-\partial \Delta, M \times M-\Delta) \subset(M \times M$, $M \times M-\Delta$ ) is a homotopy equivalence (Lemma 3.6).

Thus we get a commutative diagram of isomorphisms

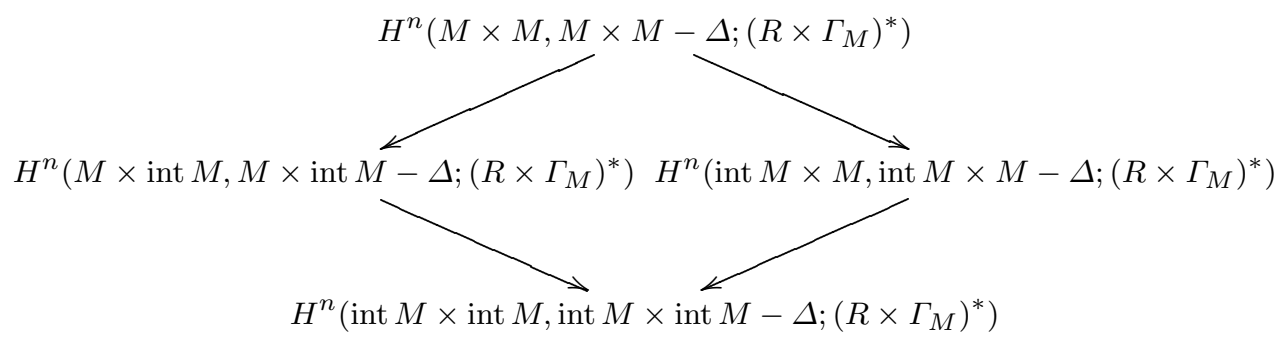

(the upper arrows are induced by excision maps) and denote by

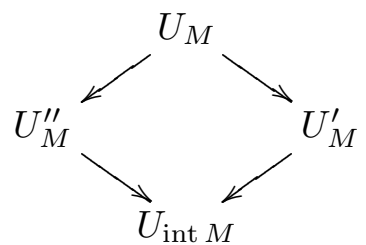

the elements corresponding to the Thom class $U_{\operatorname{int}} M \in H^{n}$ (int $M \times \operatorname{int} M$, $\left.\operatorname{int} M \times \operatorname{int} M-\Delta ;\left(R \times \Gamma_{M}\right)^{*}\right)\left(\right.$ Lemma 3.4). Let $\widetilde{U}_{M}^{\prime}, \widetilde{U}_{M}^{\prime \prime}, \widetilde{U}_{M}$ denote the restrictions of these classes to $H^{n}(\operatorname{int} M \times(M, \partial M)), H^{n}((M, \partial M) \times \operatorname{int} M)$, $H^{n}((M \times M), \partial(M \times M)-\partial \Delta)$ respectively.

LEMMA 3.8. We have

$$
\left\langle\widetilde{U}_{M}^{\prime}, \overline{1} \times z_{M}\right\rangle=1
$$

(here $\overline{1} \in H_{0}(\operatorname{int} M ; R)$ is the unit, and $z_{M} \in H_{n}\left(M, \partial M ; \Gamma_{M}\right)$ is the fundamental class). 
Pr o of. Consider the commutative diagram

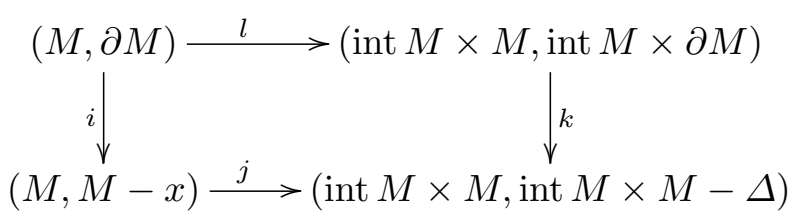

where $l(y)=j(y)=(x, y), x \in \operatorname{int} M$ fixed. Now

$$
\begin{aligned}
\left\langle\widetilde{U}_{M}^{\prime}, \overline{1} \times z_{M}\right\rangle & =\left\langle k^{*}\left(U_{M}^{\prime}\right), l_{*}\left(z_{M}\right)\right\rangle=\left\langle U_{M}^{\prime}, k_{*} l_{*}\left(z_{M}\right)\right\rangle=\left\langle U_{M}^{\prime}, j_{*} i_{*}\left(z_{M}\right)\right\rangle \\
& =\left\langle j^{*}\left(U_{M}^{\prime}\right), z_{M, x}\right\rangle=\left\langle j^{*}\left(U_{\text {int } M}^{\prime}\right), z_{\operatorname{int} M, x}\right\rangle=1,
\end{aligned}
$$

where the last equality follows from Lemma 3.4.

We will use the following notation: for $a \in H_{*} M$ and $x \in H^{*} M$ we denote the corresponding elements by $\bar{a} \in H_{*}(\operatorname{int} M)$ and $\bar{x} \in H^{*}(\operatorname{int} M)$.

Lemma 3.9. Let $M$ be a compact $n$-manifold. Then for any $x \in H^{p}(M ; R)$ and $a \in H_{p}(M ; R)$,

$$
\left\langle\widetilde{U}_{M}^{\prime}, \bar{a} \times\left(x \cap z_{M}\right)\right\rangle=(-1)^{p n}\langle x, a\rangle .
$$

Proof. We have

$$
\begin{aligned}
\left\langle\widetilde{U}_{M}^{\prime}, \bar{a} \times\left(x \cap z_{M}\right)\right\rangle & =\left\langle\widetilde{U}_{M}^{\prime},(\overline{1} \cap \bar{a}) \times\left(x \cap z_{M}\right)\right\rangle \\
& =\left\langle\widetilde{U}_{M}^{\prime},(\overline{1} \times x) \cap\left(\bar{a} \times z_{M}\right)\right\rangle \\
& =\left\langle\widetilde{U}_{\mathrm{int} M} \cup(\overline{1} \times \bar{x}), \bar{a} \times z_{M}\right\rangle \\
& =\left\langle\widetilde{U}_{\operatorname{int} M} \cup(\bar{x} \times \overline{1}), \bar{a} \times z_{M}\right\rangle \\
& =\left\langle\widetilde{U}_{M}^{\prime} \cup(\bar{x} \times 1), \bar{a} \times z_{M}\right\rangle \\
& =\left\langle\widetilde{U}_{M}^{\prime},(\bar{x} \times 1) \cap\left(\bar{a} \times z_{M}\right)\right\rangle \\
& =(-1)^{p n}\left\langle\widetilde{U}_{M}^{\prime},(\bar{x} \cap \bar{a}) \times\left(1 \cap z_{M}\right)\right\rangle \\
& =(-1)^{p n}\langle\bar{x}, \bar{a}\rangle\left\langle\widetilde{U}_{M}^{\prime}, \overline{1} \times z_{M}\right\rangle \\
& =(-1)^{p n}\langle\bar{x}, \bar{a}\rangle=(-1)^{p n}\langle x, a\rangle .
\end{aligned}
$$

Lemma 3.10. Let $M$ be a compact $n$-manifold. Then for any $x \in H^{p}(M$, $\partial M ; R)$ and $a \in H_{p}(M, \partial M ; R)$,

$$
\left\langle\widetilde{U}_{M}^{\prime \prime}, a \times\left(x \cap z_{M}\right)\right\rangle=(-1)^{p n}\langle x, a\rangle,
$$

where $x \cap z_{M}$ is regarded as an element of $H_{n-p}\left(\operatorname{int} M ; \Gamma_{M}\right)=H_{n-p}\left(M ; \Gamma_{M}\right)$. 
Proof. We have

$$
\begin{aligned}
\left\langle\widetilde{U}_{M}^{\prime \prime}, a \times\left(x \cap z_{M}\right)\right\rangle & =\left\langle\widetilde{U}_{M}^{\prime \prime},(1 \cap a) \times\left(x \cap z_{M}\right)\right\rangle \\
& =\left\langle\widetilde{U}_{M}^{\prime \prime},(1 \times x) \cap\left(a \times z_{M}\right)\right\rangle \\
& =\left\langle\widetilde{U}_{M},(1 \times x) \cap\left(a \times z_{M}\right)\right\rangle \\
& =\left\langle\widetilde{U}_{M} \cup(1 \times x), a \times z_{M}\right\rangle \\
& =\left\langle\widetilde{U}_{M} \cup(x \times 1), a \times z_{M}\right\rangle \\
& =\left\langle\widetilde{U}_{M},(x \times 1) \cap\left(a \times z_{M}\right)\right\rangle \\
& =(-1)^{p n}\left\langle U_{M},(x \cap a) \times\left(1 \cap z_{M}\right)\right\rangle \\
& =(-1)^{p n}\langle x, a\rangle\left\langle\widetilde{U}_{M}^{\prime}, \overline{1} \times z_{M}\right\rangle \\
& =(-1)^{p n}\langle x, a\rangle .
\end{aligned}
$$

A map $g: M \rightarrow N$ between two $n$-manifolds $M, N$ is called orientation true if each $\alpha \in \pi_{1} M$ preserves a local orientation of $M$ iff $g \alpha \in \pi_{1} N$ preserves a local orientation of $N$ (see [O]). Notice that then any isomorphism of $R$-modules $\phi_{x}: \Gamma_{M}(x) \rightarrow \Gamma_{N}(g x)$ (for fixed $x$ ) admits a unique extension to a morphism of local systems $\phi: \Gamma_{M} \rightarrow \Gamma_{N}$ covering $g$. For $R=\mathbb{Z}$ (the integers) there are exactly two such morphisms. In the next sections we will associate with each orientation true map $g$ a morphism $\phi$ obtained from one of the above two morphisms induced by the unique ring homomorphism $\mathbb{Z} \rightarrow R$.

4. The Lefschetz Theorem. In this section we give two extensions of the coincidence index and Lefschetz number onto pairs of maps $f, g$ : $(M, \partial M) \rightarrow(N, \partial N)$ where $M, N$ are compact $n$-manifolds and $g$ is orientation true. They generalize the classical cases: $M, N$ closed oriented [V] and oriented [BS]. In the first approach we assume $g(\partial M) \subset \partial N$ and we drop a similar assumption on $f$. Then we assume $f(\partial M) \subset \partial N$. The indices thus obtained may be different as the following simple example illustrates. Let $f, g:\left(D^{n}, S^{n-1}\right) \rightarrow\left(D^{n}, S^{n-1}\right)$ be maps of degree $k, l$ respectively. If we require that only $g$ preserves $S^{n-1}$ then $f$ is homotopic to the constant map and we obtain the index $(-1)^{n} l$. If we change the roles of $f$ and $g$ the index obtained equals $k$. We will show that in general the difference between these two indices is equal to the index of the restrictions $f_{\mid}, g_{\mid}: \partial M \rightarrow \partial N$. In particular, these two indices are equal if $M$ is closed.

First let us focus on the first generalization. We consider $f, g: M \rightarrow N$ where $g$ is orientation true and $g(\partial M) \subset \partial N$. Since $f$ is homotopic to a map into int $N$ and any two such deformations are homotopic in int $N$, we may assume that $f(M) \subset \operatorname{int} N$. 
We define the index of the pair $f, g$ as the image of the fundamental class $z_{M} \in H_{n}\left(M, \partial M ; \Gamma_{M}\right)$ under the sequence of homomorphisms

$$
\begin{aligned}
H_{n}\left(M, \partial M ; \Gamma_{M}\right) \stackrel{d_{*}^{\prime}}{\rightarrow} & H_{n}\left(M \times(M, \partial M) ; R \times \Gamma_{M}\right) \\
& \stackrel{(f \times g)_{*}}{\longrightarrow} H_{n}\left(\operatorname{int} N \times(N, \partial N) ; R \times \Gamma_{N}\right) \stackrel{\left\langle\widetilde{U}_{N}^{\prime}, \cdot\right\rangle}{\longrightarrow} R
\end{aligned}
$$

and set $\operatorname{ind}^{\prime}(f, g)=\left\langle\widetilde{U}_{N}^{\prime},(f \times g)_{*} d_{*}^{\prime}\left(z_{M}\right)\right\rangle$. This is an invariant with respect to homotopies $f_{t}, g_{t}$ satisfying $g_{t}(\partial M) \subset \partial N$. Moreover, $\operatorname{ind}^{\prime}(f, g)$ $\neq 0$ implies $f(x)=g(x)$ for an $x \in M$. Its sign depends on the choice of a morphism of local systems compatible with $g$.

We are going to define a suitable Lefschetz number.

Consider the homomorphisms

$$
\begin{aligned}
H^{q}(N ; R) \stackrel{f^{*}}{\longrightarrow} H^{q}(M ; R) \stackrel{D_{M}}{\longrightarrow} H_{n-q}\left(M, \partial M ; \Gamma_{M}\right) & \stackrel{g_{*}}{\longrightarrow} H_{n-q}\left(N, \partial N ; \Gamma_{N}\right) \stackrel{D_{N}^{-1}}{\longrightarrow} H^{q}(N ; R)
\end{aligned}
$$

and define $\theta_{q}^{\prime}=D_{N}^{-1} g_{*} D_{M} f^{*}(q=0, \ldots, n)$.

From now on we assume that $R$ is a field. We define the Lefschetz number of the pair $f, g$ by

$$
L^{\prime}(f, g)=\sum_{q=0}^{n}(-1)^{q} \operatorname{tr} \theta_{q}^{\prime}
$$

where the right side denotes the alternating sum of the traces of the endomorphisms $\theta_{q}$ of the finite-dimensional vector spaces $H^{p}(N ; R)$.

We are going to prove

TheOREM 4.1 (normalization). Let $f, g: M \rightarrow N$ be a pair of maps between compact $n$-manifolds with $g$ orientation true and $g(\partial M) \subset \partial N$. Then

$$
L^{\prime}(f, g)=\operatorname{ind}^{\prime}(f, g) .
$$

In the proof we will follow [V] (Chapter 6).

Notice that in the definition of $L^{\prime}(f, g)$ we may consider the sequences

$$
\begin{aligned}
H^{q}(\operatorname{int} N ; R) \stackrel{f^{*}}{\longrightarrow} H^{q}(M ; R) \stackrel{D_{M}}{\longrightarrow} & H_{n-q}\left(M, \partial M ; \Gamma_{M}\right) \\
& \stackrel{g_{*}}{\longrightarrow} H_{n-q}\left(N, \partial N ; \Gamma_{N}\right) \stackrel{D_{N}^{-1}}{\longrightarrow} H^{q}(\operatorname{int} N ; R)
\end{aligned}
$$

since we may assume that $f(M) \subset \operatorname{int} N$.

First we fix homogeneous bases of linear spaces over $R$ :

$a_{i} \in H_{*}(N ; R), \quad a_{i}^{\prime} \in H_{*}\left(N, \partial N ; \Gamma_{N}\right), \quad x_{i} \in H^{*}(N ; R), \quad x_{i}^{\prime} \in H^{*}\left(N, \partial N ; \Gamma_{N}^{*}\right)$

such that:

- the bases $\left\{a_{i}\right\},\left\{x_{i}\right\}$ are dual via the Kronecker index,

- the bases $\left\{a_{i}^{\prime}\right\},\left\{x_{i}^{\prime}\right\}$ are dual via the Kronecker index, and

- $D_{N}\left(x_{i}\right)=a_{i}^{\prime}$. 
Similarly we fix bases

$b_{i} \in H_{*}(M ; R), b_{i}^{\prime} \in H_{*}\left(M, \partial M ; \Gamma_{M}\right), y_{i} \in H^{*}(M ; R), y_{i}^{\prime} \in H^{*}\left(M, \partial M ; \Gamma_{M}^{*}\right)$ (here $\left.D_{M}\left(y_{i}\right)=b_{i}^{\prime}\right)$. Suppose that the homomorphisms

$f^{*}: H^{*}(\operatorname{int} N ; R) \rightarrow H^{*}(M ; R), \quad g^{*}: H^{*}\left(N, \partial N ; \Gamma_{N}^{*}\right) \rightarrow H^{*}\left(M, \partial M ; \Gamma_{M}^{*}\right)$

are given by $f^{*}\left(x_{j}\right)=\sum_{k} \gamma_{j k} y_{k}$ and $g^{*}\left(x_{j}^{\prime}\right)=\sum_{i} \beta_{j i} y_{i}^{\prime}$. Then the dual homomorphisms

$f_{*}: H_{*}(M ; R) \rightarrow H_{*}(\operatorname{int} N ; R), \quad g_{*}: H_{*}\left(M, \partial M ; \Gamma_{M}\right) \rightarrow H_{*}\left(N, \partial M ; \Gamma_{N}\right)$

are given by $f_{*}\left(b_{i}\right)=\sum_{k} \gamma_{k i} a_{k}$ and $g_{*}\left(b_{i}^{\prime}\right)=\sum_{j} \beta_{j i} a_{j}^{\prime}$.

Lemma 4.2 (cf. $[\mathrm{V} ; 6.10])$. For fixed $p$ the following equality holds in $H_{n}\left(\operatorname{int} N \times(N, \partial N) ; R \times \Gamma_{N}\right)$ :

$$
\sum_{i} a_{i} \times g_{*} D_{M} f^{*}\left(\bar{x}_{i}\right)=\sum_{j}(f \times g)_{*}\left(b_{j} \times b_{j}^{\prime}\right)
$$

where $i$ runs over the set $I_{p}=\left\{i: \operatorname{dim} a_{i}=p\right\}$ and $j$ runs over the set $J_{p}=\left\{j: \operatorname{dim} b_{j}=p\right\}$.

Proof. It is enough to prove the similar equality $\sum_{i} a_{i} \times g_{*} D_{M} f^{*}\left(x_{i}\right)=\sum_{j}(f \times g)_{*}\left(b_{j} \times b_{j}^{\prime}\right) \quad$ in $H_{n}\left(N \times(N, \partial N) ; R \times \Gamma_{N}\right)$, where $f$ is considered as a map into $N$.

Consider $g_{*} D_{M} f^{*}\left(x_{i}\right) \in H_{n-p}\left(N, \partial N ; \Gamma_{N}\right)$. Since

$$
\begin{aligned}
\left\langle x_{j}^{\prime}, g_{*} D_{M} f^{*}\left(x_{i}\right)\right\rangle & =\left\langle g^{*}\left(x_{j}^{\prime}\right), D_{M} f^{*}\left(x_{i}\right)\right\rangle=\left\langle\sum_{k} \beta_{j k} y_{k}^{\prime}, \sum_{l} \gamma_{i l} b_{l}^{\prime}\right\rangle \\
& =\sum_{k, l} \beta_{j k} \gamma_{i l}\left\langle y_{k}^{\prime}, b_{l}^{\prime}\right\rangle=\sum_{k} \beta_{j k} \gamma_{i k},
\end{aligned}
$$

we get

$$
g_{*} D_{M} f^{*}\left(x_{i}\right)=\sum_{j, k} \beta_{j k} \gamma_{i k} a_{j}^{\prime} .
$$

Now

$$
\sum_{i} a_{i} \times g_{*} D_{M} f^{*}\left(x_{i}\right)=\sum_{i}\left(a_{i} \times\left(\sum_{j, k} \beta_{j k} \gamma_{i k} a_{j}^{\prime}\right)\right)=\sum_{i, j, k} \beta_{j k} \gamma_{i k}\left(a_{i} \times a_{j}^{\prime}\right) .
$$

On the other hand, 


$$
\begin{aligned}
\sum_{j}(f \times g)_{*}\left(b_{j} \times b_{j}^{\prime}\right) & =\sum_{j} f_{*}\left(b_{j}\right) \times g_{*}\left(b_{j}^{\prime}\right) \\
& =\sum_{j}\left(\left(\sum_{i} \gamma_{i j} a_{i}\right) \times\left(\sum_{s} \beta_{s j} a_{s}^{\prime}\right)\right) \\
& \left.=\sum_{j, i, s} \gamma_{i j} \beta_{s j}\left(a_{i} \times a_{s}^{\prime}\right)\right),
\end{aligned}
$$

which implies our lemma.

LEMMA 4.3 (cf. [V; 6.11]). Under the above notations,

$$
d_{*}^{\prime}\left(z_{M}\right)=\sum_{j \in J}(-1)^{\left|b_{j}\right| \cdot\left|b_{j}^{\prime}\right|} b_{j} \times b_{j}^{\prime}
$$

where $|b|$ denotes the dimension of $b \in H_{*}(M ; R)$ and $J=\bigcup J_{p}$.

Proof. We rewrite the proof of (6.11) in [V]:

$$
\begin{aligned}
\left\langle y_{k} \times y_{l}^{\prime}, d_{*}^{\prime}\left(z_{M}\right)\right\rangle & =\left\langle y_{k} \cup y_{l}^{\prime}, z_{M}\right\rangle=(-1)^{\left|y_{k}\right| \cdot\left|y_{l}^{\prime}\right|}\left\langle y_{l}^{\prime} \cup y_{k}, z_{M}\right\rangle \\
& =(-1)^{\left|y_{k}\right| \cdot\left|y_{l}^{\prime}\right|}\left\langle y_{l}^{\prime}, y_{k} \cap z_{M}\right\rangle=(-1)^{\left|b_{k}\right| \cdot\left|b_{l}^{\prime}\right|}\left\langle y_{l}^{\prime}, b_{k}^{\prime}\right\rangle \\
& =(-1)^{\left|b_{k}\right| \cdot\left|b_{l}^{\prime}\right|} \delta_{k l}=(-1)^{\left|b_{k}\right| \cdot\left|b_{k}^{\prime}\right|} \delta_{k l} .
\end{aligned}
$$

Proof of Theorem 4.1. Fix $r=0, \ldots, n$. Then

$$
\begin{aligned}
\operatorname{tr} \theta_{r}^{\prime} & =\sum_{i \in I_{r}}\left\langle\theta_{r}^{\prime}\left(\bar{x}_{i}\right), \bar{a}_{i}\right\rangle \\
& =\sum_{i \in I_{r}}\left\langle D_{N}^{-1} g_{*} D_{M} f^{*}\left(\bar{x}_{i}\right), \bar{a}_{i}\right\rangle \\
& =\sum_{i \in I_{r}}(-1)^{n r}\left\langle\widetilde{U}_{N}^{\prime}, \bar{a}_{i} \times g_{*} D_{M} f^{*}\left(\bar{x}_{i}\right)\right\rangle \\
& =\sum_{j \in I_{r}}(-1)^{n r}\left\langle\widetilde{U}_{N}^{\prime},(f \times g)_{*}\left(b_{j} \times b_{j}^{\prime}\right)\right\rangle .
\end{aligned}
$$

Now

$$
\begin{aligned}
L(f, g) & =\sum_{r=0}^{n}(-1)^{r} \operatorname{tr} \theta_{r}^{\prime} \quad \text { (by the above equality) } \\
& =\sum_{r=0}^{n}(-1)^{r} \sum_{j \in I_{r}}(-1)^{n r}\left\langle\widetilde{U}_{N}^{\prime},(f \times g)_{*}\left(b_{j} \times b_{j}^{\prime}\right)\right\rangle \\
& =\left\langle\widetilde{U}_{N}^{\prime},(f \times g)_{*}\left(\sum_{j \in J}(-1)^{\left|b_{j}\right| \cdot\left|b_{j}^{\prime}\right|} b_{j} \times b_{j}^{\prime}\right)\right\rangle \quad(4.3) \\
& =\left\langle\widetilde{U}_{N}^{\prime},(f \times g)_{*}\left(d_{*}^{\prime}\left(z_{M}\right)\right)\right\rangle=\operatorname{ind}^{\prime}(f, g) .
\end{aligned}
$$


Corollary 4.4. If $f, g: M \rightarrow N$ where $g$ is orientation true and $g(\partial M) \subset \partial N$ then $L^{\prime}(f, g) \neq 0$ implies that $f$ and $g$ have a coincidence.

Now we are going to define an alternative index and Lefschetz number. We consider a pair $f, g: M \rightarrow N$ where $f(\partial M) \subset \partial N$ and $g$ is orientation true. The definitions will be similar to the previous ones but the symmetry is not full ( $g$ is orientation true in both cases) hence we will sketch the proof of the second version of the Lefschetz theorem.

Consider a pair $f, g: M \rightarrow N$ where $f(\partial M) \subset \partial N$ and $g$ is orientation true. We define the coincidence index of this pair as the image of the fundamental class $z_{M} \in H_{n}\left(M, \partial M ; \Gamma_{M}\right)$ under the sequence of homomorphisms

$$
\begin{aligned}
H_{n}\left(M, \partial M ; \Gamma_{M}\right) \stackrel{d_{*}^{\prime \prime}}{\longrightarrow} & H_{n}\left((M, \partial M) \times \operatorname{int} M ; R \times \Gamma_{M}\right) \\
& \stackrel{(f \times g)_{*}}{\longrightarrow} H_{n}\left((N, \partial N) \times \operatorname{int} N ; R \times \Gamma_{N}\right) \stackrel{\left\langle\widetilde{U}_{N}^{\prime \prime}, \cdot\right\rangle}{\longrightarrow} R
\end{aligned}
$$

and set $\operatorname{ind}^{\prime \prime}(f, g)=\left\langle\widetilde{U}_{N}^{\prime \prime},(f \times g)_{*} d_{*}^{\prime \prime}\left(z_{M}\right)\right\rangle$. This is an invariant with respect to homotopies $f_{t}, g_{t}$ satisfying $f_{t}(\partial M) \subset \partial N$. Moreover, $\operatorname{ind}^{\prime \prime}(f, g) \neq 0$ implies $f(x)=g(x)$ for an $x \in M$.

Now we define the corresponding Lefschetz number. We consider the homomorphisms

$$
\begin{aligned}
& H^{q}(N, \partial N ; R) \stackrel{f^{*}}{\rightarrow} H^{q}(M, \partial M ; R) \stackrel{D_{M}}{\longrightarrow} H_{n-q}\left(M ; \Gamma_{M}\right) \\
& \stackrel{g_{*}}{\rightarrow} H_{n-q}\left(N ; \Gamma_{N}\right) \stackrel{D_{N}^{-1}}{\longrightarrow} H^{q}(N, \partial N ; R)
\end{aligned}
$$

and set $\theta_{q}^{\prime \prime}=D_{N}^{-1} g_{*} D_{M} f^{*}(q=0, \ldots, n)$.

We define the second Lefschetz number as

$$
L^{\prime \prime}(f, g)=\sum_{q=0}^{n}(-1)^{q} \operatorname{tr} \theta_{q}^{\prime \prime}
$$

Theorem 4.5 (normalization). Let $f, g: M \rightarrow N$ be a pair of maps between compact $n$-manifolds with $g$ orientation true and $f(\partial M) \subset \partial N$. Then

$$
L^{\prime \prime}(f, g)=\operatorname{ind}^{\prime \prime}(f, g) .
$$

Notice that in the definition of $L^{\prime \prime}(f, g)$ we may consider the sequences

$$
\begin{aligned}
H^{q}(N, \partial N ; R) \stackrel{f^{*}}{\rightarrow} H^{q}(M, \partial M ; R) \stackrel{D_{M}}{\longrightarrow} H_{n-q}\left(\operatorname{int} M ; \Gamma_{M}\right) \\
\stackrel{g_{*}}{\rightarrow} H_{n-q}\left(\operatorname{int} N ; \Gamma_{N}\right) \stackrel{D_{N}^{-1}}{\longrightarrow} H^{q}(N, \partial N ; R)
\end{aligned}
$$

since we may assume that $g(M) \subset \operatorname{int} N$. 
First we fix homogeneous bases of linear spaces over $R$ :

$a_{i} \in H_{*}(N, \partial N ; R), a_{i}^{\prime} \in H_{*}\left(N ; \Gamma_{N}\right), x_{i} \in H^{*}(N, \partial N ; R), x_{i}^{\prime} \in H^{*}\left(N ; \Gamma_{N}^{*}\right)$ such that:

- the bases $\left\{a_{i}\right\},\left\{x_{i}\right\}$ are Kronecker dual,

- the bases $\left\{a_{i}^{\prime}\right\},\left\{x_{i}^{\prime}\right\}$ are Kronecker dual, and

- $D_{N}\left(x_{i}\right)=a_{i}^{\prime}$.

Similarly we fix bases

$b_{i} \in H_{*}(M, \partial M ; R), b_{i}^{\prime} \in H_{*}\left(M ; \Gamma_{M}\right), y_{i} \in H^{*}(M, \partial M ; R), y_{i}^{\prime} \in H^{*}\left(M ; \Gamma_{M}^{*}\right)$ (here $\left.D_{M}\left(y_{i}\right)=b_{i}^{\prime}\right)$.

Suppose that the homomorphisms

$$
f^{*}: H^{*}(N, \partial N ; R) \rightarrow H^{*}(M, \partial M ; R), \quad g^{*}: H^{*}\left(N ; \Gamma_{N}^{*}\right) \rightarrow H^{*}\left(M ; \Gamma_{M}^{*}\right)
$$

are given by $f^{*}\left(x_{j}\right)=\sum_{k} \gamma_{j k} y_{k}$ and $g^{*}\left(x_{j}^{\prime}\right)=\sum_{i} \beta_{j i} y_{i}^{\prime}$. Then the dual homomorphisms

$$
f_{*}: H_{*}(M, \partial M ; R) \rightarrow H_{*}(N, \partial N ; R), \quad g_{*}: H_{*}\left(M ; \Gamma_{M}\right) \rightarrow H_{*}\left(N ; \Gamma_{N}\right)
$$

are given by $f_{*}\left(b_{i}\right)=\sum_{k} \gamma_{k i} a_{k}$ and $g_{*}\left(b_{i}^{\prime}\right)=\sum_{j} \beta_{j i} a_{j}^{\prime}$.

Lemma 4.6 (cf. $[\mathrm{V} ; 6.10])$. For fixed $p$ the following equality holds in $H_{n}\left((N, \partial N) \times N ; R \times \Gamma_{N}\right)$ :

$$
\sum_{i} a_{i} \times g_{*} D_{M} f^{*}\left(x_{i}\right)=\sum_{j}(f \times g)_{*}\left(b_{j} \times b_{j}^{\prime}\right)
$$

where $i$ runs over the set $I_{p}=\left\{i: \operatorname{dim} a_{i}=p\right\}$ and $j$ runs over the set $J_{p}=\left\{j: \operatorname{dim} b_{j}=p\right\}$.

LEMMA 4.7 (cf. $[\mathrm{V} ; 6.11])$. Under the above notations, $d_{*}^{\prime}\left(z_{M}\right)=$ $\sum_{j \in J}(-1)^{\left|b_{j}\right| \cdot\left|b_{j}^{\prime}\right|} b_{j} \times b_{j}^{\prime}$ where $|b|$ denotes the dimension of $b \in H_{*}(M ; R)$.

Proof of Theorem 4.5. Fix $r=0, \ldots, n$. Then

$$
\begin{aligned}
\operatorname{tr} \theta_{r}^{\prime \prime} & =\sum_{i \in I_{r}}\left\langle\theta_{r}^{\prime \prime}\left(x_{i}\right), a_{i}\right\rangle \\
& =\sum_{i \in I_{r}}\left\langle D_{N}^{-1} g_{*} D_{M} f^{*}\left(x_{i}\right), a_{i}\right\rangle \\
& =\sum_{i \in I_{r}}(-1)^{n r}\left\langle\widetilde{U}_{N}^{\prime \prime}, a_{i} \times g_{*} D_{M} f^{*}\left(x_{i}\right)\right\rangle \\
& =\sum_{j \in J_{r}}(-1)^{n r}\left\langle\widetilde{U}_{N}^{\prime \prime},(f \times g)_{*}\left(b_{j} \times b_{j}^{\prime}\right)\right\rangle .
\end{aligned}
$$


Now

$$
\begin{aligned}
L^{\prime \prime}(f, g) & =\sum_{r=0}^{n}(-1)^{r} \operatorname{tr} \theta_{r}^{\prime \prime} \quad \text { (by the above equality) } \\
& =\sum_{r=0}^{n}(-1)^{r} \sum_{j \in J_{r}}(-1)^{n r}\left\langle\widetilde{U}_{N}^{\prime \prime},(f \times g)_{*}\left(b_{j} \times b_{j}^{\prime}\right)\right\rangle \\
& =\left\langle\widetilde{U}_{N}^{\prime \prime},(f \times g)_{*}\left(\sum_{j \in J}(-1)^{\left|b_{j}\right| \cdot\left|b_{j}^{\prime}\right|} b_{j} \times b_{j}^{\prime}\right)\right\rangle \\
& \left.=\left\langle\widetilde{U}_{N}^{\prime \prime},(f \times g)_{*}\left(d_{*}^{\prime \prime}\left(z_{M}\right)\right)\right\rangle=\operatorname{ind}^{\prime \prime}(f, g)\right\rangle .
\end{aligned}
$$

Corollary 4.8. If $f, g: M \rightarrow N$ where $g$ is orientation true and $f(\partial M) \subset \partial N$ then $L^{\prime \prime}(f, g) \neq 0$ implies that $f, g$ have a coincidence.

Now we are going to show a theorem connecting the above two Lefschetz numbers. We notice (cf. [Bd; VI.9.1]) that the image of the fundamental class $z_{M} \in H_{n}\left(M, \partial M ; \Gamma_{M}\right)$ under the connecting homomorphism of the pair $(M, \partial M)$ is the fundamental class of the boundary: $\partial_{*}\left(z_{M}\right)=z_{\partial M} \in$ $H_{n-1}\left(\partial M ; \Gamma_{M}\right)$.

Theorem 4.9. Let $f, g:(M, \partial M) \rightarrow(N, \partial N), g$ orientation true. Then $L^{\prime}(f, g)-L^{\prime \prime}(f, g)=L(\partial f, \partial g)$.

Pr o of. Consider the commutative (up to sign) diagram

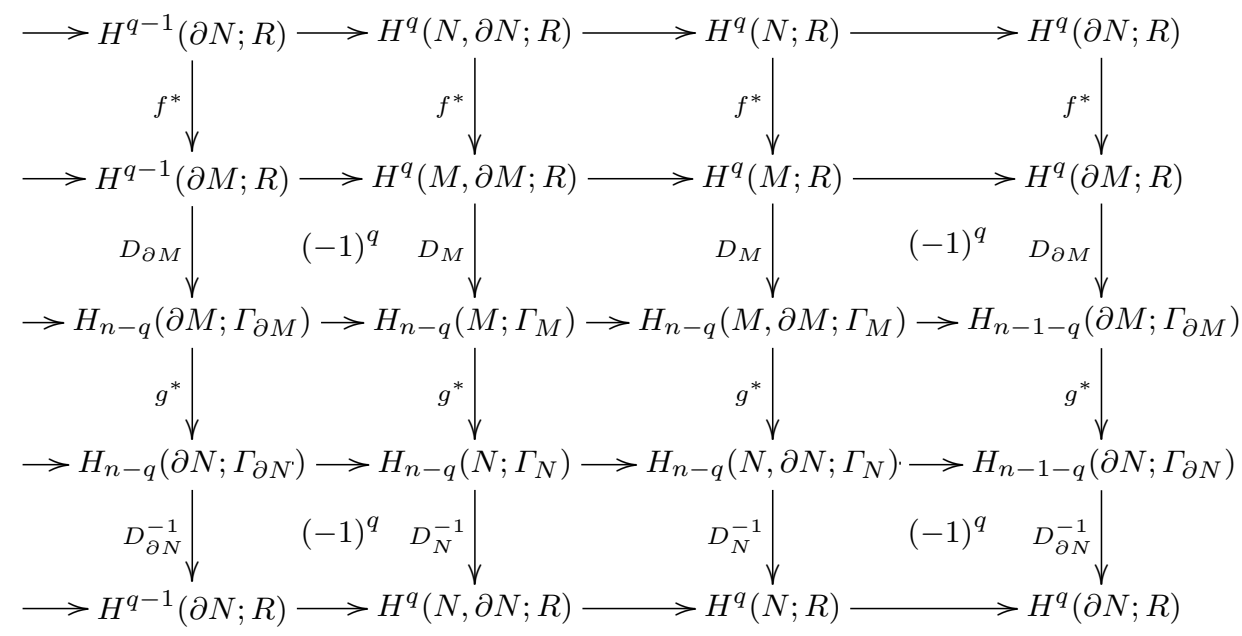

Composing the vertical arrows we get a self-map of the long exact sequence 


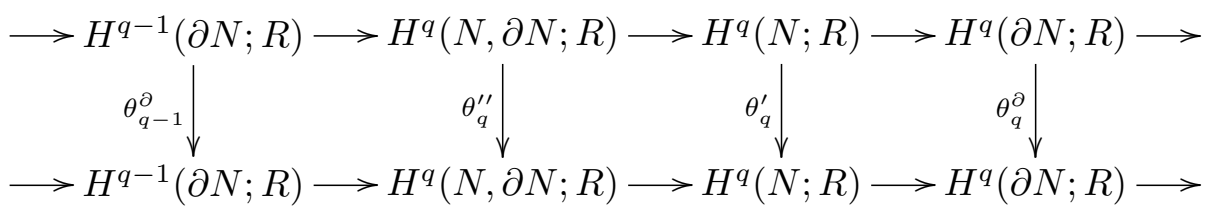

which implies $\sum_{q=0}^{\infty}(-1)^{q}\left[\operatorname{tr} \theta_{q}^{\prime \prime}-\operatorname{tr} \theta_{q}^{\prime}+\operatorname{tr} \theta_{q}^{\partial}\right]=0$, hence

$$
\sum_{q=0}^{\infty}(-1)^{q} \operatorname{tr} \theta_{q}^{\prime \prime}-\sum_{q=0}^{\infty}(-1)^{q} \operatorname{tr} \theta_{q}^{\prime}+\sum_{q=0}^{\infty}(-1)^{q} \operatorname{tr} \theta_{q}^{\partial}=0
$$

and finally

$$
L^{\prime \prime}(f, g)-L^{\prime}(f, g)+L(\partial f, \partial g)=0 .
$$

The Lefschetz coincidence number for manifolds with boundary in the oriented case was introduced in $[\mathrm{M}]$. Notice that $\Lambda(f, g)$ defined in that paper is equal to our $(-1)^{n} L^{\prime \prime}(g, f)$.

The above theorem implies that in the closed case both Lefschetz numbers $L^{\prime}(f, g)$ and $L^{\prime \prime}(f, g)$ are equal and it would be natural to expect that in this case this number satisfies $L(f, g)=(-1)^{n} L(g, f)$ (as in the oriented case). We will show that this equality is true if $g$ is the identity map. However, we will give examples showing that it is not true in general.

Definition 4.10. Let $f: M \rightarrow M$ be an orientation true map between closed $n$-manifolds. We define the degree of $f$ as the natural number $k$ satisfying $f_{*}\left(z_{M}\right)=k \cdot z_{N}$.

We denote it by $\operatorname{deg}(f)$. The sign of this number depends on the choice of a morphism of the local systems. However, in the case of coverings there is a natural morphism.

Lemma 4.11. If $p: \widetilde{M} \rightarrow M$ is a $k$-fold covering then $\operatorname{deg}(p)=k$.

Pr o of. Consider the commutative diagram

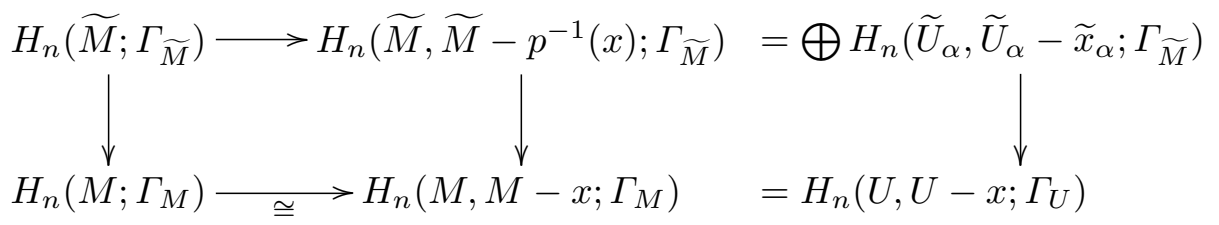

and notice that each fundamental class $z_{\alpha} \in H_{n}\left(\widetilde{U}_{\alpha}, \widetilde{U}_{\alpha}-\widetilde{x}_{\alpha} ; \Gamma_{\widetilde{M}}\right)$ is sent by the rightmost vertical arrow to $z_{U, x}$. 
Corollary 4.12. If $p_{M}: \widetilde{M} \rightarrow M$ and $p_{N}: \widetilde{N} \rightarrow N$ are $k$-fold coverings and the diagram

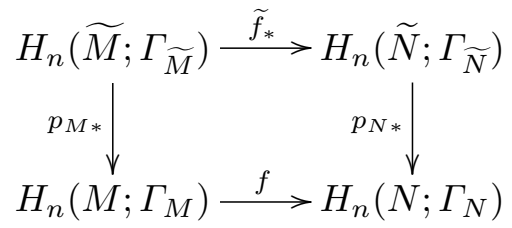

commutes then $\operatorname{deg}(f)=\operatorname{deg}(\widetilde{f})$.

Pr o o f. Indeed,

$$
\begin{aligned}
k \cdot \operatorname{deg}(f) & =\operatorname{deg}\left(p_{M}\right) \cdot \operatorname{deg}(f)=\operatorname{deg}\left(f p_{M}\right)=\operatorname{deg}\left(p_{N} \widetilde{f}\right) \\
& =\operatorname{deg}\left(p_{N}\right) \cdot \operatorname{deg}(\widetilde{f})=k \cdot \operatorname{deg}(\widetilde{f}) .
\end{aligned}
$$

ExAMPLE 4.13. Let $\widetilde{f}_{k}: S^{2} \rightarrow S^{2}$ be an odd map $\left(\widetilde{f}_{k}(-x)=-\widetilde{f}_{k}(x)\right)$ of degree $k$ ( $k$ must then be an odd number). This map induces a map $f_{k}: \mathbb{R} P^{2} \rightarrow \mathbb{R} P^{2}$. By the above corollary $\operatorname{deg}\left(f_{k}\right)=\operatorname{deg}\left(\widetilde{f}_{k}\right)=k$. On the other hand, $f_{k}$ induces an isomorphism of fundamental groups and hence is orientation true. Let us fix two odd integers $k, l$ and compute the Lefschetz number $L\left(f_{k}, f_{l}\right)$ (with rational coefficients $R=\mathbb{Q}$ ). Since $\mathbb{R} P^{2}$ is $\mathbb{Q}$-acyclic, only the sequence

$$
H^{0}\left(\mathbb{R} P^{2} ; \mathbb{Q}\right) \stackrel{f_{k}^{*}}{\rightarrow} H^{0}\left(\mathbb{R} P^{2} ; \mathbb{Q}\right) \rightarrow H_{2}\left(\mathbb{R} P^{2} ; \Gamma\right) \stackrel{f_{l}^{*}}{\rightarrow} H_{2}\left(\mathbb{R} P^{2} ; \Gamma\right) \rightarrow H^{0}\left(\mathbb{R} P^{2} ; \mathbb{Q}\right)
$$

may have a non-zero contribution to $L\left(f_{k}, f_{l}\right)$. Thus $L\left(f_{k}, f_{l}\right)=\operatorname{deg}\left(f_{l}\right)=l$. But on the other hand, $L\left(f_{l}, f_{k}\right)=\operatorname{deg}\left(f_{k}\right)=k$.

EXAMPLE 4.14. The following example shows that $L(f, g) \neq 0$ does not imply $L(g, f) \neq 0$. Let $p, c: S^{2} \rightarrow \mathbb{R} P^{2}$ where $p$ is the projection and $c$ is a constant map. It is easy to see that $L(p, c)=0$ but $L(c, p)=2$.

Despite the above examples, we do have the following positive result:

Proposition 4.15. Let $f: M \rightarrow M$ be an orientation true map of a closed $n$-manifold. Then $L(\mathrm{id}, f)=(-1)^{n} L(f$, id $)$.

Proof. Let us denote by $\left(f_{q}, R\right),\left(f^{q}, R\right),\left(f_{q}, \Gamma_{M}\right)$ the self-homomorphisms induced by $f$ on $H_{q}(M ; R), H^{q}(M ; R)$ and $H_{q}\left(M ; \Gamma_{M}\right)$ respectively. Then certainly $\operatorname{tr}\left(f^{q}, R\right)=\operatorname{tr}\left(f_{q}, R\right)$.

By the definition given at the beginning of this section,

$$
L(f, \mathrm{id})=\sum_{q=0}^{\infty}(-1)^{q} \operatorname{tr}\left(f^{q}, R\right), \quad L(\mathrm{id}, f)=\sum_{q=0}^{\infty}(-1)^{q} \operatorname{tr}\left(f_{n-q}, \Gamma_{M}\right) .
$$

Since $(-1)^{q}=(-1)^{n}(-1)^{n-q}$, it remains to show that $\sum_{q=0}^{\infty}(-1)^{q} \operatorname{tr}\left(f_{q}, R\right)$ $=\sum_{q=0}^{\infty}(-1)^{q} \operatorname{tr}\left(f_{q}, \Gamma_{M}\right)$. 
Let $K$ be a cell structure of $M$. Recall that $H_{*}(M ; \Gamma)\left(\Gamma=R\right.$ or $\left.\Gamma_{M}\right)$ may be obtained from the chain complex $\left(C_{i}, \partial_{i}\right)$ where $C_{i}=H_{i}\left(K^{i}, K^{i-1} ; \Gamma\right)$ with suitable boundary operators $\partial_{i}$ [Wh]. These boundary operators may be different in the two cases considered but certainly, by excision, $H_{i}\left(K^{i}, K^{i-1} ; \Gamma\right)$ does not depend on $\Gamma$. Let $f_{i}: C_{i} \rightarrow C_{i}$ be the induced maps at the chain level. Now we recall that the Lefschetz number of a chain map and of the induced homology map are equal. Thus $\sum_{i=0}^{\infty}(-1)^{i} \operatorname{tr}\left(f_{i}\right)=\sum_{i=0}^{\infty}(-1)^{i} \operatorname{tr}\left(f_{i}, \Gamma\right)$. Since the left hand side of this equality does not depend on the boundary operator, the right hand sides are equal in the two cases: $\Gamma=R$ and $\Gamma=\Gamma_{M}$, giving the desired equality.

Remarks. 1) More explicit computations of $L(f, g)$ have been done for maps $f, g$ between compact surfaces with $g$ orientation true in [GO]. These computations rely basically on Lemma 4.3 or more precisely on its dual which is Proposition 2.3 of [G1].

2 ) It is no surprise that the numbers $L(f, g)$ and $L(g, f)$ may be different. Notice that in our definition of $L(f, g)$ the homology homomorphism induced by the map $(f, g)$ has values in the group $H_{n}\left(M \times M ; R \times \Gamma_{M}\right)$ where the coefficients are not symmetric in the two coordinates. In fact, $L(f, g)$ and $L(g, f)$ are related to two different geometric situations. More specifically, the Lefschetz coincidence number is the sum of the indices of all coincidence points (we may assume that their number is finite). To compute these indices, we first fix a local orientation at a coincidence base point $x_{0} \in M$ and a local orientation at $y_{0}=f\left(x_{0}\right)=g\left(x_{0}\right) \in N$ (compare the definition of semi-index in the next section). Let $x_{1}$ be another coincidence point. We join these two points with a path $\gamma$ and we translate the local orientation from $x_{0}$ to $x_{1}$ along $\gamma$. To get the contribution of the coincidence point $x_{1}$ to $L(f, g)$ and $L(g, f)$ we translate the local orientation from $y_{0}$ to $y_{1}=f\left(x_{1}\right)=g\left(x_{1}\right)$ along the path $g(\gamma)$ or $f(\gamma)$ respectively. If $x_{0}$ and $x_{1}$ do not belong to the same Nielsen class then these paths may not be homotopic and may give different local orientations.

5. Coincidence index and semi-index. In this section we will define a local coincidence index for a pair of maps $f, g: M \rightarrow N$ where $M, N$ are (possibly non-compact) $n$-manifolds without boundary, the coincidence set is compact, and the map $g$ is orientation true. We will also consider homotopies $F, G: M \times I \rightarrow N$ with $\Phi(F, G)=\{(x, t) \in M \times I: F(x, t)=G(x, t)\}$ compact. We will call them $\Phi$-compact pairs of maps and $\Phi$-compact pairs of homotopies respectively. For any clopen subset $C \subset \Phi(f, g)$ we will define the coincidence index generalizing the index from the previous section (for $M, N$ closed, $C=\Phi(f, g)$ ). We will show that for any Nielsen class the absolute value of this index equals the coincidence semi-index from $[\mathrm{DJ}],[\mathrm{Je}]$. 
Let $U \subset M$ be an open subset such that $U \cap \Phi(f, g)=C$ is compact.

Definition 5.1. The coincidence index of $C$, denoted by ind $(f, g ; C)$, is the image of $z_{M, C}$ by the composition

$$
\begin{aligned}
H_{n}\left(M, M-C ; \Gamma_{M}\right) & =H_{n}\left(U, U-C ; \Gamma_{M}\right) \\
& \stackrel{(f, g)_{*}}{\longrightarrow} H_{n}\left(N \times N, N \times N-\Delta ; R \times \Gamma_{N}\right) \stackrel{\left\langle U_{N}^{\prime}, \cdot\right\rangle}{\longrightarrow} R .
\end{aligned}
$$

The above definition is correct (does not depend on the choice of $U$ ) and it is easy to see that it is homotopy invariant: for any $\Phi$-compact homotopy $F, G: M \times I \rightarrow N$ and any clopen set $C \subset \Phi(F, G)$, ind $\left(F_{t}, G_{t} ; C_{t}\right)$ does not depend on $t$.

Now we recall the definition of the semi-index [DJ], [Je].

A pair of maps $f, g: M \rightarrow N$ will be called transverse if any coincidence point $x_{0} \in \Phi(f, g)$ admits a neighbourhood $x_{0} \in U \subset N$ such that $f$ is constant on $U$ while the restriction $g_{\mid U}: U \rightarrow g(U)$ is a homeomorphism. Any pair of maps is homotopic to a transverse pair ([Je; 1.1]). Two coincidence points $x_{0}, x_{1} \in \Phi(f, g)$ are called Nielsen related if there exists a path $\omega$ joining them in $M$ such that $f \omega$ and $g \omega$ are homotopic in $N$ as paths with fixed ends. This relation splits the coincidence set into Nielsen classes.

Assume that $A$ is a Nielsen class of a transverse pair $f, g: M \rightarrow N$. Since $A$ is compact and discrete, $A$ is finite. Let $\omega$ be a path joining the points $x_{0}, x_{1} \in A$ and establishing the Nielsen relation between them and let $U$ be a neighbourhood of $x_{0}$ where $f$ is constant and $g$ is a homeomorphism. Let us fix a generator $\alpha_{0} \in H_{n}\left(U, U-x_{0} ; \mathbb{Z}\right)=H_{n}\left(M, M-x_{0} ; \mathbb{Z}\right)=\mathbb{Z}$ and let $\alpha_{t} \in H_{n}(M, M-\omega(t) ; \mathbb{Z})$ be its translation along $\omega$. On the other hand, $\beta_{0}=g_{*}\left(\alpha_{0}\right) \in H_{n}\left(g(U), g(U)-g\left(x_{0}\right) ; \mathbb{Z}\right)=H_{n}\left(N, N-g\left(x_{0}\right) ; \mathbb{Z}\right)$ is also a generator. Let $\beta_{t}=H_{n}(N, N-g \omega(t) ; \mathbb{Z})$ be its translation along $g \omega$. We say that $\omega$ establishes the $R$-relation between $x_{0}$ and $x_{1}$ if $g_{*} \alpha_{1}=-\beta_{1} \in$ $H_{n}\left(N, N-g\left(x_{1}\right) ; \mathbb{Z}\right)$ (we then write $\left.x_{0} R x_{1}\right)$.

Let us write

$$
A=\left\{a_{1}, b_{1}, \ldots, a_{k}, b_{k} ; c_{1}, \ldots, c_{s}\right\}
$$

where $a_{i} R b_{i}(i=1, \ldots, k)$ but $c_{i} R c_{j}$ for no $i \neq j$. Finally, we define the semi-index of the class $A$ as $\mid$ ind $\mid(f, g ; A)=s$. In [DJ] it was proved that the above definition is correct (i.e. the number $s$ is the same for any presentation of the class $A$ as above) and that this semi-index is a homotopy invariant (in particular, if $|\operatorname{ind}|(f, g ; A) \neq 0$ then the class $A$ cannot disappear during any homotopy).

The following lemma gives an interpretation of the coincidence index (from Section 4) at a transverse coincidence point. In the rest of this section $R=\mathbb{Z}$ (the integers). 
Lemma 5.2. Let $x_{0}$ be a transverse coincidence point of $f, g: M \rightarrow N$, $g$ be orientation true and let $U$ be a neighbourhood of $x_{0}$ where $g$ is a homeomorphism and $f$ is constant. Let $g_{*}$ denote the composition of isomorphisms

$$
\begin{aligned}
& H_{n}\left(M, M-x_{0} ; \Gamma_{M}\right)=H_{n}\left(U, U-x_{0} ; \Gamma_{M}\right) \\
& \quad \stackrel{\left(g_{\mid U}\right)_{*}}{\longrightarrow} H_{n}\left(g(U), g(U)-g\left(x_{0}\right) ; \Gamma_{N}\right)=H_{n}\left(N, N-g\left(x_{0}\right) ; \Gamma_{N}\right) .
\end{aligned}
$$

Then $g_{*}\left(z_{M, x_{0}}\right)=\left(\operatorname{ind}\left(f, g ; x_{0}\right)\right) z_{N, g\left(x_{0}\right)}$.

Pr o of. Consider the commutative diagram

$$
\begin{gathered}
H_{n}\left(U, U-x_{0} ; \Gamma_{U}\right) \stackrel{(f, g)_{*}}{\longrightarrow} H_{n}\left(N \times N, N \times N-\Delta ; Z \times \Gamma_{N}\right) \\
H_{n}\left(N, N-g\left(x_{0}\right) ; \Gamma_{N}\right)
\end{gathered}
$$

where the vertical arrow is induced by the inclusion $y \rightarrow\left(f\left(x_{0}\right), y\right)$. Since $z_{N, g\left(x_{0}\right)}$ is a generator, we may write $g_{*}\left(x_{M, x_{0}}\right)=r \cdot z_{N, g\left(x_{0}\right)}$. Since $g$ is a homeomorphism near $x_{0}$, we have $r=+1$ or -1 . Finally,

$$
\begin{aligned}
\operatorname{ind}\left(f, g ; x_{0}\right) & =\left\langle U_{N},(f, g)_{*} z_{M, x_{0}}\right\rangle=\left\langle U_{N},\left(i_{f\left(x_{0}\right)}\right)_{*} g_{*}\left(z_{M, x_{0}}\right)\right\rangle \\
& =\left\langle\left(i_{f\left(x_{0}\right)}\right)^{*} U_{N}, r \cdot z_{N, g\left(x_{0}\right)}\right\rangle=r\left\langle\left(i_{f\left(x_{0}\right)}\right)^{*} U_{N}, z_{N, g\left(x_{0}\right)}\right\rangle=r .
\end{aligned}
$$

Lemma 5.3. Let $f, g: M \rightarrow N$ be a transverse pair of maps with $g$ orientation true. Let $x_{0}, x_{1} \in \Phi(f, g)$ be Nielsen related. Then

$$
x_{0} R x_{1} \quad \text { iff } \quad \operatorname{ind}\left(f, g ; x_{0}\right)=-\operatorname{ind}\left(f, g ; x_{1}\right) .
$$

Proof. Let $\omega$ be a path establishing the Nielsen relation between $x_{0}$ and $x_{1}$. We will show that $\omega$ does not establish the $R$-relation iff ind $\left(f, g ; x_{0}\right)$ $=\operatorname{ind}\left(f, g ; x_{1}\right)$.

Let us fix a continuous family of embeddings $\sigma_{t}: \Delta_{n} \rightarrow M$ such that $\omega(t) \in \sigma_{t}\left(\right.$ int $\left.\Delta_{n}\right)$ and coefficients $\gamma_{t} \in \Gamma\left(\sigma_{t}\left(v_{0}\right)\right)$ satisfying $\left[\gamma_{t} \cdot \sigma_{t}\right]=$ $z_{M, \omega(t)} \in H_{n}\left(M, M-\omega(t) ; \Gamma_{M}\right)$. These singular simplices $\sigma_{t}$ may be chosen so small that $g \sigma_{0}$ and $g \sigma_{1}$ are also embeddings. We do not loose generality assuming that ind $\left(f, g ; x_{0}\right)=+1$. Then $g_{*}\left(z_{M, x_{0}}\right)=z_{N, g\left(x_{0}\right)}$, in other words, $z_{N, g\left(x_{0}\right)}=\left[\phi\left(\gamma_{0}\right) \cdot g \sigma_{0}\right] \in H_{n}\left(N, N-g\left(x_{0}\right) ; \Gamma_{N}\right)\left(\phi: \Gamma_{M} \rightarrow \Gamma_{N}\right.$ denotes a fixed morphism associated with the orientation true map $g$ ). It remains to show that $z_{N, g\left(x_{1}\right)}=\left[\phi\left(\gamma_{1}\right) \cdot g \sigma_{1}\right] \in H_{n}\left(N, N-g\left(x_{1}\right) ; \Gamma_{N}\right)$ iff $\omega$ does not establish the $R$-relation.

Consider a continuous family of embeddings $\tau_{t}: \Delta_{n} \rightarrow N$ and coefficients $\delta_{t} \in \Gamma\left(\tau_{t}\left(v_{0}\right)\right)$ such that $g \omega(t) \in \operatorname{int} \tau_{t}, \delta_{0}=\phi\left(\gamma_{0}\right), \tau_{0}=g \sigma_{0}, \tau_{1}\left(v_{0}\right)=$ $g \sigma_{1}\left(v_{0}\right)$. Then $\left[\delta_{0} \cdot \tau_{0}\right]=z_{N, g\left(x_{0}\right)} \in H_{n}\left(N, N-g\left(x_{0}\right) ; \Gamma_{N}\right)$ implies $\left[\delta_{1} \cdot \tau_{1}\right]=$ $z_{N, g\left(x_{1}\right)} \in H_{n}\left(N, N-g\left(x_{1}\right) ; \Gamma_{N}\right)$

It remains to find out when

$$
\left[\delta_{1} \cdot \tau_{1}\right]=\left[\phi\left(\gamma_{1}\right) \cdot g \sigma_{1}\right] \in H_{n}\left(N, N-g\left(x_{1}\right) ; \Gamma_{N}\right) .
$$


If the simplices $\sigma_{t}$ and $\tau_{t}$ are chosen sufficiently small then the paths $t \rightarrow \mathbb{Z} \tau_{t}\left(v_{0}\right)$ and $t \rightarrow g \sigma_{t}\left(v_{0}\right)$ are homotopic, hence $\delta_{0}=\phi\left(\gamma_{0}\right)$ implies $\delta_{1}=\phi\left(\gamma_{1}\right)$. Thus (5.4) holds iff $\left[\tau_{1}\right]=\left[g \sigma_{1}\right] \in H_{n}\left(N, N-g\left(x_{1}\right) ; \mathbb{Z}\right)$. But the last equality means exactly that $\omega$ does not establish the $R$-relation.

Theorem 5.5. Let $A$ be a Nielsen class of the pair $f, g: M \rightarrow N$ (where $g$ is orientation true $)$. Then $|\operatorname{ind}(f, g ; A)|=|\operatorname{ind}|(f, g ; A)$.

Proof. Since both sides are homotopy invariant, we may assume that $f, g$ is a transverse pair. Fix a presentation

$$
A=\left\{a_{1}, b_{1}, \ldots, a_{k}, b_{k} ; c_{1}, \ldots, c_{s}\right\} .
$$

Now $5.2 \operatorname{implies} \operatorname{ind}\left(f, g ; a_{i}\right)=-\operatorname{ind}\left(f, g ; b_{i}\right)$ and

$$
\operatorname{ind}\left(f, g ; c_{1}\right)=\ldots=\operatorname{ind}\left(f, g ; c_{s}\right) \quad(=+1 \text { or }-1) \text {. }
$$

Thus $|\operatorname{ind}(f, g ; A)|=s=|\operatorname{ind}|(f, g ; A)$.

Finally, let us sketch how one may obtain an index via obstruction theory. We will not require here $g$ to be orientation true, but we will consider only sets $C \subset \Phi(f, g)$ which are Nielsen classes. To simplify the formulations we only deal with maps $f, g: M \rightarrow N$ between closed manifolds.

For any Nielsen coincidence class $F \subset \Phi(f, g)$ we will define an index which is either an integer or an element of $\mathbb{Z}_{2}$.

Proposition 5.6. Given $f, g$ as above, the obstruction to deforming $f, g$ to a coincidence free pair of maps lies in $H^{n}(M ; \mathbb{Z}[\pi])$ where $\pi=\pi_{1}(N)$ and the local coefficient system is given by $\alpha \theta=\operatorname{sign}\left(g_{\#}(\alpha)\right) f_{\#}(\alpha) \theta g_{\#}(\alpha)^{-1}$ for $\alpha \in \pi_{1}(M)$ and $\theta \in \pi$.

Proof. The pair $f, g$ is homotopic to a coincidence free pair iff the following diagram admits a lift which makes it homotopy commutative:

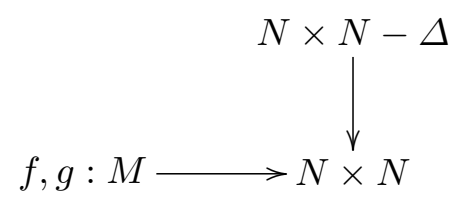

Now the result follows from Theorem 3.1 of $[\mathrm{FH}]$ and obstruction theory.

Proposition 5.7. The group $H^{n}(M ; \mathbb{Z}[\pi])$ is isomorphic to a sum of $\mathbb{Z}$ 's and $\mathbb{Z}_{2}$ 's where the summands are indexed by the Reidemeister classes.

Proof. By Poincaré duality (see [W1]), we have $H^{n}(M ; \mathbb{Z}[\pi]) \approx$ $H_{0}(M, \widetilde{Z}[\pi])$ where the homology is with the local coefficient system given by the action

$$
\alpha \circ \theta=\operatorname{sign}(\alpha) \operatorname{sign}\left(g_{\#}(\alpha)\right) f_{\#}(\alpha) \theta g_{\#}(\alpha)^{-1} .
$$


But $H_{0}$ is just the quotient of $\mathbb{Z}[\pi]$ by this action. It turns out that this quotient is either $\mathbb{Z}$ or $\mathbb{Z}_{2}$; it is $\mathbb{Z}_{2}$ if and only if there exists an $\alpha$ such that $f_{\#}(\alpha) \theta g_{\#}(\alpha)^{-1}=\theta$ and $\operatorname{sign}(\alpha) \operatorname{sign}\left(g_{\#}(\alpha)\right)=-1$. So the result follows.

Definition 5.8. Given a Nielsen coincidence class $F$, let $[\theta]$ be the Reidemeister class which corresponds to $F$. We define $i(f, g ; F)$ to be the coefficient of $[\theta] \in H_{0}(M ; \widetilde{Z}[\pi])$ which corresponds to the obstruction to deforming $f, g$ to a coincidence free pair.

More details about this definition can be found in [G1].

Remarks. 1) The index of a Nielsen class $F$ can also be defined as above in the situation where $f, g: K \rightarrow M$ are two continuous maps from a complex $K$ to a manifold (see [G2]).

2) If $g$ is orientation true then all the indices are integers.

3) The classes which have index in $\mathbb{Z}_{2}$ are called defective in [DJ] and in [G1].

6. Coincidence producing maps. It is an immediate consequence of the classical Lefschetz fixed point theorem that any compact acyclic ANR has the fixed point property [B]. Here we prove a similar result for coincidences. We recall [BS] that a map $g: M \rightarrow N$ is called a coincidence producing map if any $f: M \rightarrow N$ has a coincidence with $g$.

TheOREM 6.1 (cf. [BS; 7.1]). If any of the closed manifolds $M, N$ is $R$-acyclic (i.e. $H^{i}(X ; R)=0$ for $\left.i>0\right)$ over a field $R$ then any orientation true map $g: M \rightarrow N$ with $\operatorname{deg}(g) \neq 0$ is coincidence producing.

Proof. Let $f: M \rightarrow N$. By the $R$-acyclicity assumption only the composition

$$
\theta_{0}: H^{0}(N ; R) \stackrel{f^{*}}{\longrightarrow} H^{0}(M ; R) \stackrel{D_{M}}{\longrightarrow} H_{n}\left(M ; \Gamma_{M}\right) \stackrel{g_{*}}{\rightarrow} H_{n}\left(N ; \Gamma_{N}\right) \stackrel{D_{N}^{-1}}{\longrightarrow} H_{0}(N ; R)
$$

may have a non-zero contribution to the Lefschetz number $L(f, g)$. Now $L(f, g)=\operatorname{tr} \theta_{0}=\operatorname{deg}(g)$, hence the assumption $\operatorname{deg}(g) \neq 0$ and Corollary 4.4 give a coincidence.

\section{References}

[Bd] G. Bredon, Geometry and Topology, Grad. Texts in Math. 139, Springer, New York, 1993.

[B] R. F. Brown, The Lefschetz Fixed Point Theorem, Scott, Foresman and Co., New York, 1971.

[BS] R. F. Brown and H. Schirmer, Nielsen coincidence theory and coincidence producing maps for manifolds with boundary, Topology Appl. 46 (1992), 65-79.

[DJ] R. Dobreńko and J. Jezierski, The coincidence Nielsen theory on non-orientable manifolds, Rocky Mountain J. Math. 23 (1993), 67-85. 
[FH] E. Fadell and S. Husseini, Fixed point theory for non-simply connected manifolds, Topology 30 (1981), 53-92.

[G1] D. L. Gonçalves, Indices for coincidence classes and the Lefschetz formula for non-orientable manifolds, preprint, Mathematisches Institut, Universität Heidelberg.

[G2] -, Coincidence theory for maps from a complex into a manifold, preprint.

[GO] D. L. Gonçalves and E. Oliveira, The Lefschetz coincidence numbers for maps on compact surfaces, preprint, Department of Mathematics, UFSCAR, São Carlos.

[Je] J. Jezierski, The Nielsen coincidence theory on topological manifolds, Fund. Math. 143 (1993), 167-178.

[Ji] B. Jiang, Lectures on the Nielsen Fixed Point Theory, Contemp. Math. 14, Amer. Math. Soc., Providence, 1983.

[M] K. Mukherjea, Coincidence theory for manifolds with boundary, Topology Appl. 46 (1992), 23-39.

[O] P. Olum, Obstructions to extensions and homotopies, Ann. of Math. 52 (1950), $1-50$.

[Sp1] E. Spanier, Algebraic Topology, McGraw-Hill, New York, 1966.

[Sp2] —, Duality in topological manifolds, in: Colloque de Topologie Tenu à Bruxelles, Centre Belge de Recherches Mathématiques, 1966, 91-111.

[V] J. Vick, Homology Theory, Academic Press, New York, 1973.

[W1] C. T. C. Wall, Surgery of non-simply-connected manifolds, Ann. of Math. 84 (1966), 217-276.

[W2] -, On Poincaré complex I, ibid. 86 (1967), 213-245.

[Wh] G. W. Whitehead, Elements of Homotopy Theory, Springer, New York, 1978.

Department of Mathematics-IME

University of São Paulo

Caixa Postal 66.281-AG. Cidade de São Paulo

05389-970 São Paulo, Brasil

E-mail: dlgoncal@ime.usp.br
Department of Mathematics

University of Agriculture

Nowoursynowska 166

02766 Warszawa, Poland

E-mail: jezierski@alpha.sggw.waw.pl

Received 28 April 1995;

in revised form 25 April 1996 and 12 December 1996 